\title{
UN MODELO PARA LA EVALUACIÓN DE LA CALIDAD E-LEARNING
}

\author{
Henrik Hansson, ${ }^{5}$ Per Westman, ${ }^{6}$ \\ Eva Åström, ${ }^{7}$ Magnus Johansson ${ }^{8}$
}

\begin{abstract}
RESUMEN
La Agencia Nacional Sueca de Educación Superior llevó a cabo un estudio en el periodo 2007-2008, para adquirir conocimiento acerca de lo que constituye la calidad e-learning, y cómo puede ser evaluada dentro del esquema de un sistema nacional de asesoría de calidad.

El presente ensayo exhibe un modelo para la valuación de la citada calidad. El modelo ha evolucionado mediante el análisis de documentos legales, de redes y de proyectos de desarrollo iniciados dentro del contexto de cooperación europea, así como mediante el análisis de la forma en la cual diferentes organizaciones de evaluación nacional, y agencias encargadas de la promoción del desarrollo nacional de e-learning, lidiaron con la cuestión de la evaluación de calidad de e-learning y la educación a distancia. Estas descripciones y análisis abarcan nueve países. Los análisis indican que mientras e-learning es parte de la agenda europea, en países individuales, solo recientemente y de manera mucho más esporádica, ha surgido el tema de cómo la calidad de e-learning debe ser asesorada. En muchas organizaciones, la calidad de e-learning no parece ser un problema de interés. Un sondeo de investigaciones recientes en el área - que está incluso en este estudio- sirve como base central para el modelo de evaluación propuesto.
\end{abstract}

El modelo de la Agencia Nacional para la estimación de la calidad en e-learning «Calidad e-learning» (E-learning quality -ELQ) comprende diez aspectos de calidad los cuales, a nuestro parecer, son centrales para dicha asesoría: 1) Material/contenido; 2) Estructura/ambiente virtual; 3) Comunicación, cooperación e interactividad; 4) Evaluación del estudiante; 5) Flexibilidad y adaptabilidad; 6) Apoyo (estudiantil y del personal); 7) Cualificaciones y experiencia del personal docente; 8) Visión y liderazgo institucional; 9) Asignación de recursos; 10) Procesos y aspectos integrales.

Más allá, nuestra opinión es que la calidad e-learning debe ser asesorada desde la perspectiva de un sistema, por ejemplo, que la calidad de la educación sea determinada por todos los aspectos antes mencionados en conjunto, y por sus interrelaciones. Otra conclusión central

5 Department of Computer and Systems Sciences, Stockholm University, Forum 100, 16440 Kista, Sweden. E-mail: henrik.hansson@dsv.su.se

6 Swedish National Agency for Higher Education, Department of EvaluationBox 7851, SE-103 99 Stockholm, Sweden. E-mail: per.westman@hsv.se

7 Swedish National Agency for Higher Education, Department of Evaluation Box 7851, SE-103 99 Stockholm, Sweden. E-mail: per.westman@hsv.se

8 Swedish National Agency for Higher Education, Department of Evaluation Box 7851, SE-103 99 Stockholm, Sweden. E-mail: per.westman@hsv.se 
es que si una agencia nacional u otra organización evalúan e-learning, no es suficiente con simplemente establecer aspectos de calidad. El cuerpo evaluador también debe desarrollar y adaptar sus propios métodos de trabajo y garantizar la aptitud interna: los métodos existentes de evaluación de calidad deben ser adaptados; aspectos de calidad de e-learning deben ser integrados en sistemas de asesoría de calidad; la competencia interna y la provisión de información en el área e-learning deben ser garantizadas; los métodos de trabajo internos deben ser adaptados a las condiciones especiales y pertinentes para la asesoría de la educación $\sin$ fronteras.

Palabras clave: Educación superior, e-learning, evaluación de la calidad, modelo de evaluación, comparaciones internacionales, ICT, Educación mejorada mediante el uso de la tecnología.

\begin{abstract}
The Swedish National Agency of Higher Education conducted a study, 2007-2008, in order to develop knowledge about what constitutes quality in e-learning, and how such quality may be assessed within the framework of a national quality assurance system.

This paper presents a model for quality assessment of e-learning. The model has been developed using analyses of policy documents, networks and development projects initiated within the framework of European cooperation and analyses of how different national assessment organizations and agencies charged with promoting the national development of e-learning deal with the question of quality assessment of e-learning and distance learning. These descriptions and analyses cover nine countries. The analyses indicate that while e-learning is on the agenda in many European contexts and in individual countries, it is only recently - and much more sporadically - that the subject has been broached of how e-learning quality should be assessed. In many organizations, quality in e-learning appears to be a non-issue. A survey of current research in the area is also included in this study, and serves as the central basis for the proposed assessment model.
\end{abstract}

The National Agency's model for assessing quality in e-learning - Elearning quality (ELQ) comprises ten quality aspects which, in our view, are central to such assessments: 1) Material/ content, 2) Structure/virtual environment, 3) Communication, cooperation and interactivity, 4) Student assessment, 5) Flexibility and adaptability, 6) Support (student and staff), 7) Staff qualifications and experience, 8) Vision and institutional leadership, 9) Resource allocation, 10) The holistic and process aspect.

Further, it is our view that elearning quality must be assessed from a systems perspective, i.e. that the quality of the education is determined by all of the above aspects taken together, and by their interrelationships. Another central conclusion is that if a national agency or other organization is to assess e-learning, it is not enough simply to draw up quality aspects. The assessing body also needs to develop and adapt its own working methods and guarantee its internal competence: Existing methods of quality assessment need to be adapted; Quality aspects for elearning need to be integrated into existing quality assurance systems; Internal competence and the provision 
of information in the e-learning area need to be guaranteed; Internal working methods need to be adapted to the special conditions which apply for the assessment of borderless education.

Key words: Higher education, e-learning, quality assessment, evaluation model, international comparisons, ICT, Technology enhanced education.

Recibido: 16 de marzo de 2009

Aceptado: 17 de abril de 2009

\section{INTRODUCCIÓN}

Varias universidades, desde los años noventa, han lidiado con la evaluación de calidad y auditoría de e-learning. Sorprendentemente, pocas organizaciones asesoras de calidad han estado involucradas. En el Reino Unido, la Agencia de Asesoría de Calidad de Educación Superior formuló un marco para evaluar la calidad de la educación a distancia en 1997, y en los Estados Unidos las organizaciones CHEA y DECT han formulado criterios y puntos de referencia para la educación a distancia. En Europa, organizaciones como EFQUEL (Dondi \& Moretti, 2007) y EADTU (E-xcellence, 2007) han estado trabajando con sistemas para la evaluación de e-learning. Estos sistemas, ambos, se encuentran en periodos de prueba. También ha habido iniciativas nacionales en el desarrollo de parámetros específicos para e-learning y para la educación a distancia moderna, por ejemplo, en Suecia (Nätunivrsitetet 2003), en Noruega (NADE 2002) y en Australia (ACODE 2006). Todas estas iniciativas de sistemas, puntos de referencia y formas de evaluación, tratan e-learning como algo diferente de la educación tradicional de las ciudades universitarias. Nos encontramos en una fase en la cual la educación a distancia y la educación presencial se están mezclando para formar una educación flexible a nivel mundial. Es, por lo tanto, importante para todas las organizaciones evaluadoras, conocer los nuevos retos de e-learning. Nosotros presentamos un sistema con diez dimensiones de calidad y parámetros relacionados, los cuales deben ser integrados en los métodos de acreditación, señalización, asesoría y auditoría, para poder comprender la complejidad de los sistemas educativos actuales.

\section{OBJETIVOS Y METODOLOGÍA}

El objetivo principal de este ensayo es el de proveer una síntesis basada en la práctica e investigación internacional sobre la evaluación de la calidad de e-learning. Las preguntas de investigación principales son: ¿Qué constituye la calidad e-learning en la educación superior y, qué retos implica dicha calidad para las universidades y cuerpos evaluadores? Con este fin:

1. Se resume la situación en Suecia y en la Unión Europea, con base en documentos legales y reportes anteriores.

2. Se manda un cuestionario a cuerpos evaluadores y organizaciones relevantes en Australia, Canadá, Dinamarca, Finlandia, Países Bajos, Noruega, y el Reino Unido, donde se piden reportes importantes, documentos, URL, y personas contacto para entrevistas futuras.

3. Se resume y se analiza tanto la información como los documentos importantes recibidos de las organizaciones antes mencionadas y de la CHEA y DECT en los Estados Unidos. 
4. Se analizan los artículos de tratos realizados para e-learning, publicados del 2002 al 2007, y la literatura citada en dichos artículos.

El resultado ha sido publicado de un modo más extenso en el reporte Calidad e-learning (Agencia Nacional Sueca de Educación Superior, 2008). Hemos escogido el termino e-learning para referirnos a todos los tipos de aprendizaje basados en tecnología, tales como la educación a distancia, educación en línea, aprendizaje a distancia, aprendizaje flexible, y aprendizaje combinado. Por lo tanto, incluye gran parte de la educación en ciudades universitarias que utilizan la informática como complemento a la educación presencial.

\section{ELQ, MODELO PARA LA EVALUACIÓN DE LA CALIDAD E-LEARNING}

Basados en la información de organizaciones internacionales y, más que todo, en el análisis de investigaciones recientes de la calidad e-learning, se ha desarrollado un modelo ELQ (E-learning quality/calidad E-learning) con diez aspectos de calidad que consideramos cruciales al evaluar la calidad e-learning. Los aspectos se encuentran distribuidos en orden de importancia, pero existe una secuencia aproximada desde la parte más pequeña, los objetivos de aprendizaje, hasta una visión integral y sistemática. Esto, de hecho, también refleja las dos diversas pero complementarias fuentes de información que hemos usado en este estudio: aquellas con una perspectiva organizacional y aquellas con una perspectiva investigativa. Los aspectos de calidad son áreas temáticas, cada una con un conjunto de problemas y cuestiones e-learning específico. Para cada dimensión de calidad han sido desarrollados de tres a cuatro parámetros de calidad. Estos son recomendaciones para acciones concretas a fin de lidiar con los problemas y cuestiones identificadas a nivel institucional.

\section{Material / Contenido}

La cantidad de contenido académico disponible y producido para e-learning es enorme. Una de las cuestiones principales relacionadas con el material y el contenido es la selección y la secuencia del material y, la calidad del material usado y producido en una clase (Conolly, et al., 2005 \& Horizon report 2007). El contenidoacadémico está evolucionando de un libro impreso a multimedia interactiva, deshaciendo las barreras entre contenido, ambiente virtual y enseñanza, y, entre el aprendizaje y la interacción. Sin embargo, los estudiantes que son capaces de usar lo mejor de ambos mundos, es decir, libros impresos y recursos digitales, logran mejores resultados, ejemplificando la necesidad de competencias combinadas.

Por muchos cientos de años, el contenido académico básico ha consistido de libros impresos. En e-learning, el libro impreso sigue SIENDO de importancia, pero el contenido académico en el mundo digital es mucho más variado. De hecho, incluso los medios antiguos están siendo producidos usando nuevos medios: hoy en día, prácticamente todos los libros impresos son producidos digitalmente (Pavlik, 2004). Esto significa que el libro impreso existirá también de manera digital, con todas las características y propiedades de los medios digitales, haciéndolo fundamentalmente diferente. Hay software para poder leer el texto en voz alta al lector por medio de una computadora o de un teléfono inteligente, usando audífonos o altoparlantes. 
Otras transformaciones de los textos son ilustraciones adicionales o multimedia, dadas por el profesor, estudiante, o cualquier persona en la red. Un libro puede ser visto como una película, en vez de ser leído. El libro se enriquece con otros medios y se vuelve interactivo. Debido a este enriquecimiento, surge una necesidad de nuevos métodos de estandarización del material (Prpitsch \& Veith, 2006) para permitir su uso y reutilización en diferentes ambientes virtuales.

Ambos, el contenido de cursos nacionales e internacionales disponibles gratuitamente, se encuentran siendo desarrollados por organizaciones e individuos. UNESCO ha desarrollado la Plataforma de Entrenamiento Abierto con material libre y disponible para todos. Universidades individuales, tales como Open University en el Reino Unido y мIт Open Courseware (OCw) también dan su contenido de forma gratuita. Cuando materiales abiertos son usados, no se puede esperar una experiencia estudiantil unificada (Connolly, et al., 2005). La combinación de contenido de aprendizaje gratuito disponible y el desarrollo de estándares tienen un gran potencial para generar tanto ahorros económicos como mejoras en la calidad (Moore y Kearslye, 2005). El contenido académico electrónico incluye, cada vez más, conferencias en video. Las conferencias en video también son producidas y transmitidas por universidades, maestros individuales, y estudiantes, utilizando programas de internet sencillos y disponibles para todos como YouTube y programas de blogs de video como Wordpress o Blogger.

Hoy en día, no se da por entendido que el material académico es creado por el profesor. En muchos casos, especialmente al tratar con medios más complejos, un equipo de expertos se encuentra involucrado en dicha creación (E-xcellence 2007). En algunos casos, los estudiantes se han convertido en los creadores de su propios materiales de aprendizaje (Horizon report 2008). Esto formula preguntas relacionadas con la calidad del producto, y con el proceso de producción. Los derechos de propiedad intelectual son otra inquietud importante cuando se trata de producción esparcida y en algunos casos, colaborativa (Magjuka, et al., 2005, Kidney, et al., 2007). El reto principal no es la complejidad de los medios, es la complejidad del proceso de producción. (Horizon report 2007).

Resumen. En e-learning, el curso/material puede ser digital o escrito. Por lo tanto, la selección, producción y adaptación del contenido académico es un parámetro importante en la evaluación de la calidad. El contenido académico puede ser producido por editoriales, profesores independientes o por un grupo de desarrolladores. Al lidiar con medios digitales complejos, un equipo de expertos en producción es usualmente requerido. En algunos casos, los estudiantes se han convertido en los creadores de su propio material de aprendizaje. El reciclaje del material disponible en línea y el hecho de que los «originales» digitales no puedan ser autenticados o distinguidos fácilmente de copias, suma a la complejidad de identificar un «autor».

Estos diferentes procesos de producción formulan preguntas no solo relacionadas con la calidad del material académico, sino también acerca de los derechos de autor. 


\section{Parámetros de calidad:}

a) Reglas y guías para la selección y producción de material digital, incluyendo criterios pedagógicos y técnicos explícitos

b) Reglas y guías relacionadas con los derechos de autor

c) Conocidas e implementadas, las dos anteriores

d) Evaluación interna y mejoras subsecuentes de las tres anteriores.

\section{Estructura/ambiente virtual}

Algunas características pedagógicas útiles de un ambiente virtual incluyen maneras fáciles y estructuradas para encontrar información y para comunicarse con los compañeros y maestros. La infraestructura técnica debe ser estable, fidedigna, accesible, y amigable con el usuario (E-Xcellence 2007 y ACODE 2006).

Hoy en día, el ambiente de aprendizaje virtual para cada estudiante consiste en una gran número de herramientas usadas día a día, desde buscadores en línea, comunicación de voz por internet, mensajería instantánea, grupos de chat, foros, correo electrónico, blogs, programas de interacción social, sistema de videoconferencias digitales, hasta portafolios digitales en línea y sistemas operativos sociales (Horizon report 2007-2008). En resumen, los usuarios expertos utilizan una mezcla de programas en el mundo virtual para resolver tareas y problemas en su diario vivir, y el uso de estas herramientas incluye aprendizaje formal e informal. La tecnología social es ampliamente aceptada, permite la colaboración y amplía la presencia social (Horixon report 2008). Otro factor importante para programas populares es su facilidad de uso, su velocidad, permitir control por parte del usuario, y el ser económicos, preferiblemente gratuitos.

El ritmo al cual nuevos programas son creados y otros se vuelven obsoletos es muy veloz. Por ejemplo, las plataformas tempranas de e-learning - implementadas para facilitar e-learning a través del almacenamiento de material académico, el manejo del curso y la comunicación asincrónica basada en textos durante el curso dentro de una misma estructura - estaban basadas en sistemas administrativos de manejo desarrollados con fines empresariales. Hay muchos términos diversos para estos programas de computadora, como Sistemas de manejo del aprendizaje (LMSLearning Management Systems), Ambientes de educación virtual (VLE-Virtual Learning Environments), Sistemas de manejo de cursos (CMs-Course Management Systems), Sistemas de apoyo del aprendizaje (lss- Learning Support Systems) y Plataformas para el aprendizaje (LP-Learning Plataforms). El término plataformas para el aprendizaje, se está desarrollando hoy en día hacia un tipo de comunicación más amplia y hacia la integración de PDA y teléfonos celulares (Horizon report 2008).

Los ambientes de aprendizaje virtuales también se están desarrollando como un producto de la industria de juegos digitales, al adoptar una estrategia diferente para la interacción en línea. Los ambientes de aprendizaje basados en juegos son audiovisuales, tienen tres dimensiones y hacen énfasis en la presencia y en la comunicación sincronizada. Segunda Vida (Second Life) es utilizado de manera exitosa en la enseñanza de matemática (Caprotti; Seppälä, 2007) e idiomas. 
Situaciones y problemas de la vida real son imitados y el aprendizaje experimental, «haciendo cosas» respalda el trabajo en equipo, discusiones, y actividades de resolución de problemas. Con ambientes de aprendizaje virtuales nuevos y en vías de desarrollo, con las comunidades abiertas y en ocasiones gratuitas, y con los mundos virtuales comercialmente disponibles (Segunda Vida), la institución no se encuentra fácilmente en control de la calidad.

La selección de herramientas y los Sistemas de Manejo del Aprendizaje (LMS, por sus siglas en inglés) influyen en la interacción entre el profesor y el estudiante de un curso. La elección de LMS y programas adicionales depende, en gran parte, de la habilidad del profesor para utilizar dichos programas efectivamente, más que de la habilidad de los jóvenes estudiantes de las nuevas generaciones. Sin embargo, no todos en las nuevas generaciones han tenido la oportunidad o la motivación para desarrollar las habilidades necesarias para el uso de programas e-learning. Estudiantes de las generaciones más viejas, habitualmente necesitan más entrenamiento preparatorio en cómo usar computadoras, internet, y los programas específicos seleccionados para una clase. Este parámetro de calidad es discutido más adelante en el aspecto relacionado con el «apoyo». Al utilizar programas, sin un contrato con los vendedores, comunidades, o personas individuales, provenidos del ambiente, la institución no tiene casi control de la calidad, accesibilidad o uso del ambiente de aprendizaje. Este tema, es tratado más adelante bajo la sección denominada «políticas y liderazgo institucional».

Resumen. El ambiente digital es una de las características más dinámicas y cambiantes de e-learning, así que el mejoramiento sistemático y la actualización son necesitados en una base continua. La selección de un ambiente virtual, debería basarse en consideraciones pedagógicas y en el ambiente técnico de la institución.

\section{Parámetros de calidad:}

a) Un ambiente virtual seleccionado en base a las necesidades pedagógicas, que sea fidedigno y estable, alineado con la infraestructura técnica de la institución

b) Evaluación interna, puesta al día, y mejoramiento del anterior parámetro.

\section{Comunicación, cooperación e interactividad}

La comunicación, la cooperación y la interacción son el núcleo del aprendizaje. Una diferencia principal con la educación en una ciudad universitaria es la necesidad de un mejor planeamiento para que la comunicación se lleve a cabo (Moore \& Kearsley, 2005). La estructura comunicativa escogida para un curso e-learning particular depende de la infraestructura disponible, de las habilidades de los maestros y estudiantes y, de los objetivos del curso. La colaboración, por ejemplo, es fomentada a través de los juegos en línea, los blogs y las wikis. Dicha colaboración se puede extender a comunidades en línea abiertas al público, o puede ser protegida y solo ser accesible por los estudiantes del curso. Para tareas específicas, un ambiente cerrado puede ser la mejor opción, mientras que los ambientes abiertos añaden nuevas dimensiones y posibilidades a la discusión. El mundo digital no se encuentra delimitado por bordes físicos. Hay grandes oportunidades para cooperación y participación internacional en el desarrollo y provisión de e-learning. 
La comunicación, como parte de un curso e-learning, puede ser organizada dentro de cuatro dimensiones de tiempo y espacio (ver Tabla 1 en el apéndice). Muchos de los cursos e-learning utilizan mayormente comunicación asincrónica. De acuerdo con el reporte Horizon (2008) la colaboración explícita para crear tiendas de conocimiento colaborativo (inteligencia colectiva/ collective intelligence) será importante en e-learning en unos años.

Dos enfoques fundamentalmente diversos pueden ser identificados en el diseño de cursos e-learning. Un enfoque que puede ser llamado el «Enfoque Sócrates», se centra en el diálogo entre profesores y estudiantes. Este enfoque requiere la tecnología que aumenta y enriquece los canales de comunicación. El «contenido» del curso es construido durante los diálogos auténticos propios de cada curso. El proceso pregunta-respuesta entre los alumnos y el profesor es central. Sin embargo, no es posible para un profesor responder y estar en contacto con un gran número de alumnos, veinticuatro horas al día, siete días a la semana. La comunicación debe, por lo tanto, ser organizada de acuerdo a un contrato regulador de las horas de los profesores, los canales de comunicación utilizados, el tiempo de respuesta y el apoyo.

El otro enfoque se basa en contenido prefabricado y en actividades interactivas de e-learning. Ejemplos típicos de esto son algunos de los ambientes basados en juegos, donde la interactividad y el aprendizaje tienen lugar sin la guía de un profesor. El primer enfoque hace énfasis en el profesor, mientras que el segundo reduce el rol del maestro. Un gran número de estudiantes es viable en el segundo enfoque, mientras que el primero se enfoca en la calidad de los diálogos entre los individuos, limitando el número de estudiantes. Modelos intermedios combinan estos dos enfoques de distintas maneras.

Resumen. Los nuevos ambientes digitales de aprendizaje y sus contenidos respaldan la comunicación, la cooperación y la interactividad de maneras nuevas y diferentes. Usualmente se necesita de mayor tiempo para que estas sucedan. La transparencia de estos sistemas requiere información sobre cómo será su uso particular en el curso o programa educacional.

\section{Parámetros de calidad:}

a) Estrategia explícita para la comunicación, cooperación e interactividad de acuerdo con las necesidades pedagógicas, la tecnología disponible y los recursos humanos

b) Implementación de la anterior

c) Evaluación y mejora de las anteriores.

\section{Evaluación del estudiante}

No hay diferencia fundamental entre la evaluación en línea y la evaluación presencial. Los estudiantes tienden a responder primero los requerimientos de la evaluación, de modo que la innovación del aprendizaje debe incluir la innovación y alineamiento de la evaluación (Laurillard 2006). La evaluación en línea implica básicamente la oportunidad de incrementar los métodos de las dinámicas en grupo, el tiempo y el lugar. La posibilidad de diversificar los métodos de evaluación como simulaciones, seminarios virtuales y trabajo de grupo asincrónico, la añade 
e-learning. Para ilustrar: cuando algún material es presentado abiertamente y los estudiantes pueden hacer uso de él individualmente, una experiencia estudiantil unificada no puede esperarse (Conolly, et al., 2005). Esto conlleva un cambio radical de la manera en la cual los procesos de aprendizaje son diseñados y, por lo tanto, de cómo la evaluación es llevada a cabo. Otro ejemplo son los exámenes automatizados o semiautomatizados, los cuales son usados comúnmente (Moore \& Kearsley, 2005) y se han probado como herramientas útiles cuando son parte de una evaluación acumulativa. La evaluación en línea también ha añadido retos debido a cuestiones de seguridad, accesibilidad, e identificación (Clarke, et al., 2004, Rowe 2004). Desde la perspectiva del estudiante, la evaluación debe ser asegurada y accesible legalmente. La seguridad legal para los estudiantes se basa en una infraestructura técnica confiable, y en las respuestas rápidas de los administradores y maestros.

La institución se encuentra más a menudo preocupada por la identificación de los estudiantes y el plagio. El problema con la identificación no es nuevo; existe dentro de la educación superior una larga tradición de tareas para la casa y las cuestiones de la identificación. Una vigilancia segura en centros de aprendizajes es utilizada normalmente en otras universidades para superar el tema de la evaluación remota segura (Clarke, et al., 2004 \& Mills, 2006). El uso de evaluación sincronizada, haciendo uso de cámaras web, ID en la computadora, y escáner de huellas digitales son ejemplos de cómo superar el problema de la identificación en evaluaciones basadas en la web (Moore \& Kearsly, 2005). La identificación es mejorada, tal como lo es la validación del conocimiento adquirido, cuando se pueden arreglar diálogos en directo. Un enfoque multimodal de la evaluación provee muchas maneras en las cuales los estudiantes pueden expresar sus conocimientos, utilizando textos, producciones digitales, presentaciones orales, y discusiones orales o en grupos. Dicho enfoque también provee al profesor de abundante información, necesaria para evaluar el nivel de conocimiento y habilidades del estudiante. Sin embargo, los procedimientos de evaluación ambiciosos requieren más tiempo docente y soporte tecnológico más avanzado. La adaptación de la metodología con la declaración de Bolonia también debe ser tomada en cuenta en este contexto.

También debe ser consciente la institución de que arreglar localidades físicas para la evaluación genera, igualmente, costos adicionales para la institución y para los estudiantes. Las diferentes formas en las cuales las evaluaciones estudiantiles en línea son organizadas pueden ser categorizadas, básicamente, en términos de tiempo, sincrónicas o asincrónicas, y en términos de espacio, formal, semiformal, o informal (ver Tabla 2 en el apéndice).

Laflexibilidad puede seralineada en conflicto con las metas pedagógicas. El conflicto es básicamente entre la evaluación sincrónica y la asincrónica (ver la discusión bajo comunicación).

Resumen. Los métodos utilizados para evaluar a los estudiantes determinarán la forma en la que estos actúan hacia sus estudios, por lo que son de gran importancia pedagógica. Los sistemas de evaluación deben incentivar la creatividad, el pensamiento crítico y el conocimiento a fondo del tema que se va a tratar. En e-learning, la flexibilidad en términos de tiempo y espacio ofrece la posibilidad de ampliación. Al mismo tiempo, la flexibilidad acarrea problemas de seguridad y autenticación, $\mathrm{y}$, los procedimientos y los métodos reguladores 
deben ser puestos en práctica para asegurar la accesibilidad, la identidad del estudiante y la autenticidad de la contribución de cada individuo.

\section{Parámetros de calidad:}

a) Estrategia para evaluaciones justas, flexibles, y pedagógicamente justificadas

b) Políticas establecidas para lidiar con plagios, seguridad legal e identificación de los estudiantes

c) Implementación de las dos anteriores

d) Evaluación y mejora de las dos primeras.

\section{Flexibilidad y adaptabilidad}

Un aspecto crucial de la calidad e-learning es el grado de flexibilidad. Muchas personas tienen el deseo de aprender pero se encuentran limitadas porque tienen un horario laboral, una vida familiar, o por la localidad, la economía, el tiempo disponible u otros. Lo más flexible en el diseño de un curso es la mayor cantidad de personas que tendrán acceso a este. La flexibilidad puede ser construida de muchas maneras: horas de inicio flexibles, cursos abiertos ( tareas (los estudiantes pueden escoger y especializarse), flexibilidad en la localidad (donde se estudia), flexibilidad en el método de estudio (transmitido mediante varios canales/modos) y, la habilidad de adaptar a personas con necesidades especiales. De igual manera, la flexibilidad debe ser balanceada en contraste con la estructura (ver E-xcellence 2007, UNIQUE, QAA).

Inicialmente, la tecnología móvil no fue diseñada para propósitos educativos, pero es usada cada día más como la base para e-learning. Las nuevas generaciones de estudiantes esperan que la información sea accesible fácilmente y que la comunicación sea posible desde cualquier lugar, a cualquier hora y con cualquier persona (Horizon report 2008). Los «estudiantes nómadas» estudian a distancia, pero permanecen cerca para practicar en el puesto de trabajo o en el campo. Este tipo de e-learning es paradigmáticamente diferente del enfoque tradicional de la educación a distancia, en el cual la educación es ofrecida y es accesible a personas en áreas remotas porque no se pueden mover fácilmente de dichas áreas (Hansson \& Holmberg, 2006). La flexibilidad incrementada requiere que el contenido y las herramientas de comunicación se encuentren disponibles a través de diferentes sistemas inalámbricos y que sean independientes de hardware (Ally, 2007).

El uso del móvil puede ser tomado como una especialidad emergente entre el campo más amplio de consumo según Kukulska-Hulme (2007). Los test de uso se encuentran convencionalmente limitados a la asesoría del tiempo que toma la finalización de una tarea, en conjunto con el esfuerzo, el rendimiento, la flexibilidad y la actitud del usuario. Kukulska-Hulme (2007) argumenta que debemos movernos más allá de estos parámetros de uso (tales como accesibilidad, consistencia, confianza) y añadir «el uso pedagógico» que incluye factores tales como el control del aprendiz, la actividad del aprendiz, la motivación y la retroalimentación. El uso pedagógico es, en parte, específico para la disciplina; los estudiantes de idiomas requieren funciones diferentes que aquellas necesitadas por los estudiantes de física, por ejemplo. 
Una plataforma de aprendizaje independiente y movible, está estrechamente relacionada con el concepto de computación ubicua: «Como opuesto al paradigma del escritorio, en el cual un solo usuario conscientemente toma parte de un dispositivo individual para un propósito especializado, alguien utilizando computación ubicua toma parte en varios dispositivos y sistemas simultáneamente, en el curso de actividades ordinarias, y no necesariamente deben estar conscientes de ello».

Resumen. Características flexibles del diseño de cursos incluyen: dónde se estudia (lugar), cuándo se estudia (tiempo), el periodo durante el cual se estudia (duración), el paso al cual se estudia (tiempo completo/medio tiempo), los idiomas de instrucción y contenido, la adaptación de métodos para personas con discapacidades, el número de personas admitidas (alcance), estudios individuales o estudios en grupos. El aumento en la flexibilidad de una característica podría llegar a suprimir la flexibilidad de otra característica. La adaptación hacia grupos específicos es necesaria.

\section{Parámetros de calidad:}

a) Estrategia para aumentar las características flexibles de una educación basada en consideraciones pedagógicas y en las necesidades y exigencias de los estudiantes

b) Implementación de la anterior

c) Evaluación y mejora de las anteriores.

\section{Apoyo (estudiantil y del personal)}

Un gran número de estudios han demostrado que el apoyo es crucial para una implementación e-learning exitosa. Andersson (2007) resumió 36 ensayos de investigación discutiendo temas de apoyo en e-learning de acuerdo con cuatro categorías: Apoyo hacia los estudiantes por parte de los profesores; apoyo social para los estudiantes; apoyo del empleador; apoyo para el cuerpo docente. El artículo de Muilenburg \& Berge «Barreras Estudiantiles en el Aprendizaje en Línea: un factor de estudio analítico» (2005) reporta resultados de un estudio global de las barreras principales del aprendizaje en línea, desde la perspectiva de un estudiante. Los factores hallados fueron temas administrativos, interacción social, habilidades académicas, habilidades técnicas, la motivación del aprendiz, el tiempo y apoyo hacia los estudios, costo y acceso a internet, problemas técnicos. El apoyo oportuno y adecuado puede, según los autores, reducir significativamente la magnitud de estos problemas, más no eliminarlos totalmente.

El apoyo debe ser visto como una variable en el tiempo: apoyo para preparar a los profesores y alumnos para los estudios en línea, y apoyo durante la duración del curso. Desde nuestro punto de vista, el aspecto de calidad de los temas de apoyo incluye la eficacia del apoyo de la organización para con los estudiantes, maestros, y otro personal involucrado en los diferentes niveles: a) técnico; b) académico, incluyendo la participación de bibliotecarios; c) manejo, incluyendo una guía de estudio y, d) social, incluyendo los consejos de guías y consejeros. 
Enfatizado especialmente en los países Nórdicos (véase NADE y NSHU) se encuentra el apoyo de consejeros para la guía estudiantil. El apoyo puede ser organizado localmente o a distancia, usando comunicación sincrónica o asincrónica. El apoyo también puede ser organizado antes de tiempo en la forma de Preguntas frecuentes, y durante la duración del curso mediante el uso del correo electrónico, el chat, el teléfono u otros medios de comunicación. El tipo de apoyo que se encuentra disponible, cómo utilizarlo, a quién contactar, cómo y cuándo contactarlos, el tiempo de respuesta, y otros, así como las condiciones que aplican, todo debe ser comunicado de forma clara y consistente a los estudiantes y profesores con anterioridad.

En mayor medida, la efectividad del apoyo lo cual determina la calidad de un curso - percibida desde el punto de vista de un estudiante-, una guía de estudio, un documento básico e individual describiendo el curso en su totalidad y lo que se espera del estudiante, incluyendo las asignaciones, ayuda al aprendizaje y reduce la necesidad de apoyo en clase debido a información poco precisa. El apoyo también es importante para poder establecer una situación de trabajo sostenible para los profesores, bajo el riesgo de trabajar muchas horas o de ser responsables de muchas partes del proceso e-learning.

Resumen. El apoyo hacia estudiantes y el personal debe incluir mucho más que temas técnicos. En e-learning, el apoyo social es normalmente considerado no como igual, sino más importante. Los profesores necesitan el apoyo de bibliotecarios y de los consejeros así como de los consultores de ITC y de los administradores.

\section{Parámetros de calidad:}

a) Estrategia para el apoyo estudiantil, incluyendo apoyo técnico, administrativo y social según lo requerido

b) Estrategia para el apoyo del cuerpo docente, incluyendo técnico, ICT y competencias informáticas según lo requerido

c) Implementación de las dos anteriores

d) Evaluación y mejora de todas las anteriores.

\section{Cualificaciones y experiencia del cuerpo docente}

La necesidad de entrenamiento en servicio no es específica para la educación en línea, pero aparte del entrenamiento normal en enseñanza y de aprendizaje puede también incluir los siguientes objetivos (modified from Laurillard 2006, p. 226): crear conciencia del uso de nuevas tecnologías en su campo, de cómo los estudiantes deberían aprender a través de medios diversos, de las expectativas y, un enfoque crítico hacia las nuevas tecnologías, y el desarrollo de habilidades evaluativas para mejorar el diseño del aprendizaje.

También es importante construir nuevos modelos para el reclutamiento y la retención de personal. Según Sixl-Daniell (2004), el personal docente debe ser tecnológicamente orientado hacia hacer énfasis en la enseñanza. También, es de gran importancia que el concepto tecnológico de cómo hacerlo sea integrado con su uso pedagógico (véase Yeung, 2002). Los 
maestros no podrán, de igual manera, hacerse cargo de todos los aspectos relacionados con el desarrollo de un curso e-learning y la enseñanza per se. Deberán depender de expertos adicionales (Coolly et al., 2005). La creación de un grupo multidisciplinario, para producir cursos y materiales, es el primer paso hacia el apoyo y desarrollo de habilidades del cuerpo docente. Aparte del profesor, el equipo normalmente incluirá bibliotecarios, diseñadores de instrucción, productores multimedia y expertos ICT. Los bibliotecarios juegan un rol clave en el apoyo de los profesores y estudiantes, con fuentes de información relevantes relacionadas con la enseñanza y el aprendizaje en diferentes áreas. Conolly et al., (2005) argumentan que en cuanto a la longevidad del material y el diseño del aprendizaje, ellos mismos proveen la oportunidad «...de evaluar críticamente lo que como educadores creemos importante, y cuestionar lo que hacemos y porqué lo hacemos.»

Resumen. Las cualificaciones del personal docente y su experiencia son factores claves para el éxito. No sólo los profesores, todo el personal involucrado en e-learning debe adquirir las habilidades necesarias, y sistemáticamente poner al día sus conocimientos y estrategias.

\section{Parámetros de calidad:}

a) Estrategia para el desarrollo de las aptitudes del personal

b) Implementación de dicha estrategia

c) Evaluación y mejora de las anteriores.

\section{Visión y liderazgo institucional}

Las universidades involucradas profundamente en e-learning deben cambiar y fortalecer su manejo drásticamente a través de la organización, desde la relación directa entre el estudiante y el profesor, hasta la asignación de fondos, estrategia, y planificación (Bates 1999, Marcus 2004, Jara 2006, Paulucci \& Gambescia 2007). Los cambios son originados mediante el uso de nuevas tecnologías y el aumento de las aptitudes. Nuevos mercados, debido a la limitación de bordes geográficos, el aumento de proveedores no gubernamentales, así como al incremento en la diversidad de los estudiantes, son todos retos que deben ser abarcados.

Según Bates (1999) uno de los temas más importantes es la alineación de las políticas para e-learning con la visión general de la institución. El liderazgo organizacional debe «ser explícito acerca de a quién intenta servir, cómo y por qué» (Moore \& Kearsley, 2005) y cómo e-learning hace parte de dicha visión. Esto, sin embargo, es raramente llevado a cabo, incluso por las organizaciones con una larga tradición en el uso de e-learning. La adaptabilidad de las políticas y la planeación deben mantener el ritmo con un índice de cambio creciente en las posibilidades pedagógicas (Waysluk \& Berge, 2007). La administración debe enfocarse en la transición, ser proactiva, y actuar como un modelo, ya que los cambios en la tecnología normalmente generan situaciones caóticas (Marcus, 2004). El modelo interno de evaluación de calidad de la institución también debe ser ampliado para incluir criterios específicos de e-learning. El establecimiento de un proceso de evaluación incluirá, naturalmente, un grupo mucho más diverso de administradores de alto rango (Ellis et al., 2007), algunos de los cuales no habrán manejado enseñanza ni aprendizaje. 
Para fomentar la innovación, también es importante que la universidad tenga recursos protegidos y una estrategia de investigación clara, la aseguración de la calidad y el desarrollo de e-learning (Bates 1999, Marcus 2004, Laurillard 2006, Gaytan 2007).

Cuando diversos departamentos de una universidad son responsables de diferentes áreas del desarrollo del curso y del proceso de enseñanza, nuevas estrategias administrativas son necesarias para mantener la cohesión (Ellis et. al., 2007). Según Laurillard (2006) el paso más importante que se debe tomar es la implementación de una organización de aprendizaje, es decir, una organización que intente conducir un diálogo de aprendizaje interno que permita dicho aprendizaje a base de experiencias. Buen manejo puede, por lo tanto, ser resumido por lo siguiente: expandir el conocimiento; proveer acceso a publicaciones periódicas, de turismo y materiales de aprendizaje; compartir conocimiento; establecer grupos de aprendizaje multidisciplinario; establecer foros o animar la participación en los foros existentes, reusar el material de aprendizaje; disponer personal para el desarrollo de programas; innovar; asignar recursos protegidos y, el compromiso y tiempo del personal; establecer políticas para estándares e infraestructura, implementar; retribuir excelencia; comunicar nuevos requerimientos a los alumnos y al personal; validar; monitorear la implementación, y, actuar.

Las tecnologías de información y comunicación son parte de los conductores principales de los rápidos procesos de globalización en curso. El e-learning se convierte, cada día más, en un fenómeno internacional. Alianzas estratégicas entre universidades, compañías medianas, proveedores de ICT y otros interesados serán de gran importancia para esfuerzos e-learning sostenibles y exitosos.

Resumen. Una visión a largo plazo para e-learning debe guiar la práctica actual y establecer un objetivo común para la institución. Esta visión debe ser revisada y puesta al día regularmente. La promoción de investigación, el aseguramiento de la calidad y el desarrollo a nivel institucional generan conocimiento y experiencias cruciales para mejores actividades e-learning. La cooperación nacional, la internacional y las alianzas estratégicas son cada día más importantes. La retroalimentación, el seguimiento, y el manejo estratégico de la institución administrativa, animan y apoyan al personal y a los estudiantes que forman parte de las iniciativas e-learning.

\section{Parámetros de calidad:}

a) Un plan estratégico para e-learning con una perspectiva visionaria, incluyendo la investigación, el aseguramiento de calidad y las actividades de desarrollo; alianzas locales, nacionales e internacionales, relacionadas con objetivos a corto, mediano y largo plazo

b) Implementación y evaluación del anterior

c) Retroalimentación, seguimiento y monitoreo nacional de las tendencias internacionales y del manejo estratégico de la administración institucional.

\section{Asignación de recursos}

El tiempo del personal es, inevitablemente, el mayor costo en la enseñanza y el aprendizaje. Cuando nos movemos de una interacción cara a cara hacia un ambiente e-learning, usualmente hay un cambio dramático entre el tiempo que el personal pasa en la presentación 
al tiempo que pasa en la planeación y diseño (véase Bates, 1999 y Laurillard, 2006). En la fase de implementación, un aumento de los fondos puede ser requerido para tratar con la falta de habilidades - entrenamiento del personal, reclutamiento de personal con nuevas habilidadesasí como para la reorganización de la administración y de la infraestructura técnica (Moore \& Kearsley, 2005). Durante la creación de nuevos programas, recursos adicionales deben ser asignados al diseño del currículo y del curso. En los programas e-learning esto incluye el diseño de materiales de aprendizaje digitales y el reuso de materiales ya existentes. Con la posibilidad de reusar, los costos del e-learning pueden ser reducidos sustancialmente si la propiedad financiera y los derechos de autor se encuentran claramente establecidos (véase más en el contenido 1). El regreso de inversiones depende de la cantidad de estudiantes registrados y la sostenibilidad. La promoción de programas e-learning no necesariamente debe costar más, pero se deben enfocar otros grupos en lugar del mercadeo clásico de las ciudades universitarias. Bates (1999) también argumenta que las estrategias de financiación deben comenzar como apoyo centralizado para ayudar a los administradores de proyectos. Sin embargo, la reasignación de los recursos a la altura del curso debe ser manejada en el nivel departamental, mientras que la infraestructura y el apoyo especializado deben ser manejados en el nivel central.

La comunicación lleva tiempo y puede ser la razón principal para la extenuación a largo plazo y la pérdida de interés de los profesores a distancia. Hay, sin embargo, muy poca investigación relacionada con este fenómeno en los profesores de educación superior y es casi inexistente en e-learning (véase Hogan et al., 2007). Como el rol de un profesor e-learning es complejo, es importante proveer descripciones de trabajo claras y mantener líneas concretas de comunicación entre el personal administrativo y el personal docente (Hogan et. al., 2007). Han existido numerosos reportes acerca de la creciente carga laboral del personal que trabaja con e-learning, pero también de programas donde la suma de trabajo ha disminuido. El estudiante e-learning normalmente exige la disponibilidad del personal en las tardes y durante los fines de semana. Esto no es siempre un aspecto negativo, pero el cambio en las horas de trabajo debe ser tomado en consideración. Nuevos modelos para estimar las sumas de trabajo y un modelo financiero para conferencias virtuales y módulos interactivos deben ser puestos en práctica (Bates, 1999).

Resumen. En e-learning, los recursos deben ser reasignados desde localidades físicas (salones de conferencia, librerías, oficinas administrativas) hasta infraestructuras técnicas, organizaciones de apoyo y desarrollo del personal. En una educación combinada, ambas, la infraestructura técnica y las localidades físicas, deben ser financiadas. Las sumas de trabajo y el cambio en las horas laborales del personal también deben ser tomadas en cuenta. El desarrollo de contenido interactivo y el de conferencias en línea requieren recursos financieros específicos así como regulación de los derechos de autor. Una nueva estrategia financiera será necesaria para la propaganda de e-learning, a manera de alcanzar nuevos grupos de enfoque. 


\section{Parámetros de calidad:}

a) Una estrategia para la reasignación de los recursos existentes y la creación de nuevos recursos basados en las necesidades específicas de e-learning

b) Una estrategia y una planeación para lidiar con los cambios de las cargas de trabajo y de las horas laborales, así como con los derechos de propiedad y financieros de las conferencias virtuales individuales y otros materiales digitales

c) Implementación de las anteriores.

\section{Procesos y aspectos integrales}

E-learning consiste en componentes múltiples, por ejemplo, el software de aprendizaje, los apoyos académico y técnico, la presentación del contenido y la interacción. Los evaluadores deben reconocer que todos los componentes del programa trabajen juntos de una manera eficiente, así el sistema deberá ser efectivo (Rovai, 2003). Esta complejidad inherente a e-learning en la educación superior ha sido comúnmente descuidada (Zellwger Moser, 2007). Por lo tanto, no es sorprendente que diferentes parámetros de calidad, en muchos casos, no tengan una coherencia explícita (Ellis y Moore, 2006). Una perspectiva integral implica que todo el criterio de calidad en combinación constituye un sistema funcional. Luego, un cambio en una de las áreas temáticas debido a nuevas tecnologías, cambios en el comportamiento, u otras, usualmente, requieren ajustes de uno o más aspectos de calidad. Esto implica que en el modelo ELQ presentado aquí, todos los nueve aspectos previos están interrelacionados y constituyen un sistema. La selección de la forma como se usan las tecnologías puede ser determinada por contenido, por quién la va a enseñar y por dónde tendrá lugar el aprendizaje; y el diseño de los medios de instrucción depende del contenido, de la tecnología y de la interacción deseada (Moore y Kearsley, 2005).

Resumen. Durante la implementación de e-learning es importante adoptar un enfoque integral. Si tan solo uno de los diez aspectos no se encuentra concebido, diseñado o implementado de la manera correcta, el resultado final no será lo suficientemente bueno. Los diez aspectos hacen parte de un rompecabezas en el cual todas las piezas deben cuadrar juntas. Cuando una parte del rompecabezas cambia debido a cambios en la tecnología, al comportamiento estudiantil, a las necesidades del conocimiento, a la sociedad, la financiación o las necesidades del personal, todas las otras partes deben ser realineadas correspondientemente. Las iniciativas e-learning deben ser planeadas con el desarrollo sistemático y continuo en mente.

\section{Parámetros de calidad:}

a) Un enfoque funcional y sistemático hacia la implementación de e-learning que incluya todos los aspectos de calidad mencionados previamente: material/contenido, estructura/ ambiente virtual, comunicación, cooperación e interactividad, evaluación estudiantil, flexibilidad y adaptabilidad, apoyo (estudiantil y personal), las cualificaciones del personal y sus experiencias, visión y liderazgo institucional, asignación de recursos

b) Evaluación interna, renovación y mejora del anterior, usando un enfoque integral. 


\section{Observaciones finales}

Creemos que el modelo ELQ puede ser utilizado para resaltar y enfatizar aspectos de la educación superior donde la nueva tecnología, fundamentalmente, cambia los viejos paradigmas. Las dimensiones identificadas son de ayuda para las instituciones educativas en relación a la creación de sus políticas y la implementación de procesos, como también para la acreditación de agencias nacionales que evalúan la calidad desde una perspectiva nacional. Estudios nacionales cruzados acerca de estas dimensiones de calidad, serían muy útiles para desarrollo futuro del conocimiento de esta área.

\section{REFERENCIAS BIBLIOGRÁFICAS}

ALLY, M. Mobile Learning. The International Review of Research in Open and Distance Learning, 8 (2). 2007.

CAPROTTI, O. \& SEPPÄLÄ, M. Mathematics Education in Second Life. Department of Mathematics and Statistics University of Helsinki. Presented at 6th EDEN Open Classroom, 24-26 October 2007, Stockholm. 2007.

CLARKE, M.; BUTLER, C.; SCHMIDT-HANSEN, P.; SOMERVILLE, M. Quality assurance of distance learning: A case study at Brunel University. British Journal of Educational Technology, 35 (1). 2004.

CONNOLLY, M.; JONES, N.; O'SHEA, J. Quality assurance and e-learning: Reflection from the front line. Quality in Higher Education, 11 (1). 2005.

CHEA. Accreditation and Assuring Quality in Distance Learning. CHEA monographseries 2002.

DONDI, C. \& MORETTI, M. 2007: E-learning quality in European Universities: Different Approaches for different purposes. Retrieved from http://unique.europace.org/index.php Consultada el 15 de octubre de 2007.

HANSSON, H. \& HOLMBERG, C. Sweden: Distance education, development and competing paradigms. In: W. Zhang (Ed.). Global Perspectives: Philosophy and Practice in Distance Education (Volume Three), Beijing: China Central Radio \& Television University Press. 2006.

HANSSON, H, WESTMAN, P, ÅSTRÖM, E \& JOHANSSON, M. Aspects and criteria for evaluation of e-learning in higher education. Swedish National Agency for higher Education E-learning quality. Report 2008:11 R. Authors: 2008.

KIDNEY, G.; CUMMINGS, L.; BOHEM, A. Towards a quality assurance approach to e-learning courses. International Journal on E-Learning, 6 (1). 2007. 
KUKULSKA-HULME, A. Mobile usability in educational contexts: What have we learnt? The International Review of Research in Open and Distance Learning, 8 (2). 2007.

LAURILLARD, D. Rethinking University Teaching, a Framework for the Effective Use of Learning Technologies. Second edition. Abingdon, London: RoutledgeFalmer. 2006.

MAGJUKA, R. J.; SHI, M.; BONK, C. J. Critical design and administrative issues in online education. Online Journal of Distance Learning Administration, 8 (4). 2005.

MILLS, R. Quality assurance in distance education - towards a culture of quality: A case study of the Open University, United Kingdom (OUUK). In: B. N. Koul \& A. Kanwar (Eds). Perspectives on Distance Education. Towards a Culture of Quality. Commonwealth of learning, Vancouver. 2006.

MOORE, M. G. \& KEARSLEY, G. Distance Education a Systems View. Second edition. Thomson Wadsworth, Belmont, California. 2005.

PAVLIK, J. V. \& McINTOSH, S. Converging Media: An Introduction to Mass Communication. Boston. 2004.

PRPITSCH, C. \& VEITH, P. Content and management standards: LOM, SCORM and content packaging. In: U. D. Ehlers \& J. M. Pawlowski, (Eds). Handbook on Quality and Standardisation in E-leaning. Springer, Berlin - Heidelberg. 2006.

ROWE, N. C. Cheating in online student assessment: Beyond plagiarism. Online Journal of Distance Learning Administration, 7 (2). 2004.

\section{URL}

ACODE, 2006, http://www.acode.edu.au/projects/benchmarking.htm (Retrieved 2008-09-27). E-xcellence, http://www.eadtu.nl/e-xcellence/. (Retrieved 2007-10-10).

NADE, 2002: Kvaltietsnormer for fjernundervisning http://www.nadenff.no/files// Kvalitetsnormer.pdf. (Retrieved 2008-09-27).

Nätuniversitetet (2003). Kvalitet i IT-stödd distansutbildning. In Swedish only. http://www. nshu.se/page/2934/kvalitetskriterier.htm (Retrieved 2008-09-27).

The Horizon report. 2007 Edition. www.nmc.org/pdf/2007_Horizon_Report.pdf.

The Horizon report. 2008 Edition. www.nmc.org/pdf/2008-Horizon-Report.pdf 


\section{APÉNDICES}

Tabla 1. Tecnologías de comunicación e información relacionadas con el lugar y el tiempo

\begin{tabular}{|c|c|c|}
\hline & Lugar de prueba & Lugar diferente \\
\hline $\begin{array}{l}\text { A la misma } \\
\text { hora }\end{array}$ & $\begin{array}{l}\text { 1. Tecnología - enseñanza apoyada } \\
\text { - Demostración de programas } \\
\text { - Presentación visual de programas }\end{array}$ & $\begin{array}{l}\text { 3. Comunicación sincrónica } \\
\text { - Videoconferencias } \\
\text { - Chat/mensajería instantánea } \\
\text { - Teléfono IP } \\
\text { - Pizarra inteligente } \\
\text { - Audio chat }\end{array}$ \\
\hline $\begin{array}{l}\text { A diferentes } \\
\text { horas }\end{array}$ & $\begin{array}{l}\text { 2. Tecnología - enseñanza apoyada } \\
\text { - Estudio individual }\end{array}$ & $\begin{array}{l}\text { 4. Comunicación asincrónica } \\
\text {-Correo electrónico } \\
\text {-Foros en línea } \\
\text {-Foros auditivos } \\
\text {-Conferencias en video en línea } \\
\text {-Mensajería de texto }\end{array}$ \\
\hline
\end{tabular}

Tabla 2. Una matriz para la organización de la evaluación e-learning

\begin{tabular}{|l|l|l|l|l|l|}
\hline $\begin{array}{l}\text { Lugar de } \\
\text { evaluación }\end{array}$ & Formal * & $\begin{array}{l}\text { Semiformal ** } \\
\text { sincrónico }\end{array}$ & $\begin{array}{l}\text { Semiformal } \\
\text { asincrónico }\end{array}$ & $\begin{array}{l}\text { Informal*** } \\
\text { sincrónico }\end{array}$ & $\begin{array}{l}\text { informal } \\
\text { asincrónico }\end{array}$ \\
\hline Beneficios & $\begin{array}{l}\text { De fácil } \\
\text { identificación } \\
\text { identificación, } \\
\text { flexibilidad de } \\
\text { lugar } \\
\text { moderada }\end{array}$ & $\begin{array}{l}\text { De fácil } \\
\text { identificación, } \\
\text { flexibilidad de } \\
\text { hora y lugar } \\
\text { moderada }\end{array}$ & $\begin{array}{l}\text { Alta flexibilidad } \\
\text { de lugar. De bajo } \\
\text { costo para los } \\
\text { estudiantes, no } \\
\text { hay necesidad de } \\
\text { viajar, necesidad } \\
\text { de alojamiento }\end{array}$ & $\begin{array}{l}\text { Alta flexibilidad } \\
\text { de hora y lugar. } \\
\text { De bajo costo } \\
\text { para los } \\
\text { estudiantes, no } \\
\text { hay necesidad de } \\
\text { viajar, necesidad } \\
\text { de alojamiento }\end{array}$ \\
\hline Desventajas & $\begin{array}{l}\text { Inflexible en } \\
\text { términos de } \\
\text { hora y lugar, } \\
\text { costos } \\
\text { adicionales }\end{array}$ & $\begin{array}{l}\text { Inflexible en } \\
\text { términos de } \\
\text { hora, costos } \\
\text { adicionales }\end{array}$ & $\begin{array}{l}\text { Costos } \\
\text { adicionales }\end{array}$ & $\begin{array}{l}\text { Alta } \\
\text { identificación de } \\
\text { preocupaciones. } \\
\text { Pero, por } \\
\text { ejemplo, los } \\
\text { sistemas bancarios } \\
\text { en internet han } \\
\text { desarrollado } \\
\text { sistemas de alta } \\
\text { seguridad para la } \\
\text { identidad en este } \\
\text { medio. }\end{array}$ \\
\hline
\end{tabular}

* En la ciudad universitaria

** En localidades no gobernadas por la Universidad, pero consideradas centros de aprendizaje, embajadas, etc.

*** O independientes. Pueden ser en cualquier lugar, sólo hay restricciones técnicas tales como el uso del computador o acceso a internet. 